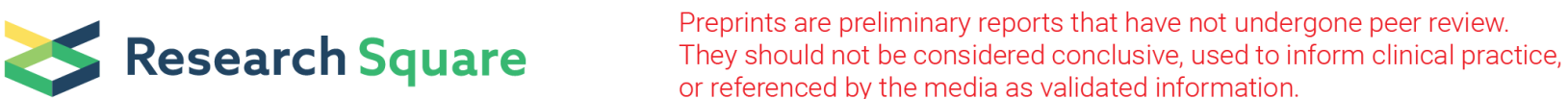 \\ Genomic insight for Algicidal activity in Rhizobium sp. (AQ_MP)
}

\section{Mili Pal}

CSIR-NEERI: National Environmental Engineering Research Institute CSIR

\section{Asifa Qureshi ( $\nabla$ a_qureshi@neeri.res.in )}

National Environmental Engineering Research Institute CSIR https://orcid.org/0000-0001-8719-4482

Hemant Purohit

CSIR-NEERI: National Environmental Engineering Research Institute CSIR

\section{Research Article}

Keywords: Harmful algal blooms, Microcystis aeruginosa, Rhizobium sp., Scanning Electron Microscopy (SEM), degradation, Microcystins (MCs)

Posted Date: May 11th, 2021

DOl: https://doi.org/10.21203/rs.3.rs-461504/v1

License: (c) (i) This work is licensed under a Creative Commons Attribution 4.0 International License. Read Full License

Version of Record: A version of this preprint was published at Archives of Microbiology on August 3rd, 2021. See the published version at https://doi.org/10.1007/s00203-021-02496-z. 


\section{Abstract}

Occurrence of Harmful Algal Blooms (HABs) creates a threat to aquatic ecosystem affecting the existing flora and fauna. Hence, the mitigation of HABs through an eco-friendly approach remains a challenge for environmentalists. The present study provides the genomic insights of Rhizobium sp. (AQ_MP), an environmental isolate that showed the capability of degrading Microcystis aeruginosa (Cyanobacteria) at laboratory scale. Genome sequence analysis of Rhizobium sp. (AQ_MP) was performed to determine the algal lysis properties and toxin degradative pathway. It is envisaged that Rhizobium sp. (AQ_MP) secreted CAZymes like Glycosyltransferases (GT), Glycoside Hydrolases (GH), polysaccharide lyases (PL), which allowed algal polysaccharide degradation (lysis) and enabled nutrient release for the subsequent growth of Rhizobium sp. (AQ_MP) Genome analysis also showed the presence of the glutathione metabolic pathway, which is the biological detoxification pathway responsible for microcystin degradation. The conserved region mlrC, a microcystin toxin degrading responsible gene, was also annotated in Rhizobium sp. (AQ_MP). This study confirmed that Rhizobium sp. (AQ_MP) harbours a wide range of crucial enzymes released for lysis of Microcystis aeruginosa (M. aeruginosa) cells and also for degradation of microcystin toxin. This study thus find promiscuity for scaling the lab based analysis to field level in future.

\section{Introduction}

Cyanobacteria are the photosynthetic organisms found in both freshwater and marine environment (Pal, $M$ et al., 2018), which can be pelagic and benthic (Wehr, J.D et al., 2015). Although cyanobacteria have few positive trends such as fixing atmospheric nitrogen but on the other hand, few species release toxic secondary metabolites such as dermatoxins, hepatotoxins, cytotoxins, and neurotoxins (Carmichael, W.W., 2001, Pearson, L., et al., 2010, Schmidt, J.R., et al., 2014). Cyanobacteria have another ability to avoid predation by grazers (Lampert, W., 1987). They can form elongated shapes, colonies and release some toxic secondary metabolites. The increased anthropogenic activities these days have increased the concentration of nitrogen and phosphate in water bodies which in the major contributor to the proliferation of algal bloom (J.R., et al., 2014). Algal bloom affects the entire water body by hindering light penetration and toxin release (Paerl, H.W., et al., 2011, Pal M., et al., 2020). The recreational activities and drinking of these water affected by algal blooms often disturb animal health (Pal M., et al., 2020). Some of these algal bloom species release lethal toxins. The Microcystis species, such as M. aeruginosa, M. fosaquae, $M$. wesenbergii, $M$. ichthyoblabe, and $M$. phertaare are the leading cause of almost $90 \%$ of the harmful algal blooms in freshwater. $M$. aeruginosa is the most commonly observed cyanobacterial species causing harmful algal blooms and releases toxin, mainly microcystins (MCs) (Kim, M., et al., 2019), which are neurotoxin and hepatotoxins. Till date, more than 90 types of microcystins are released. Still, microcystin-LR is the most abundant and highly toxic variant (Pal M., et al., 2020). Toxic mechanism of MCs is due to the disruption of cytoskeleton formation and inhibition of protein phosphatase. In humans, MCs can enter through toxin-contaminated water or diet; they can cause oxidative stress, leading to cell damage (J.R., et al., 2014). Microcystins are readily water-soluble because their log of 
octanol and water distribution ration is approximately $-1(\mathrm{pH} 7)$. The essential binding sites of microcystins to the protein phosphatase are methyl-dehydroalanine (Mdha) and 3-amino-9-methyoxy2,6,8-trimethyl-10-phenyl-4,6-decadienoic acid (ADDA) groups. Microcystin toxicity depends on the amino acid combination present at the two different positions of the peptide ring. Microcystin-LR has amino acid leucine and arginine at the variable peptide ring, and Microcystin-RR has arginine and arginine (Rinehart, K.L., et al., 1994, Corbel, S., et al., 2014).

Zhang, J., et al., 2017 reported an innate effective bacterium of Sphingopyxis sp. having microcystin degrading capability. It contained enzymes of microcystin degradation i.e. mlrA, mlrC and mlrD. Mann, A.J., et al., 2013 reported bacterium Formosa agariphila (KMM 3901T) has a broad potential of algal polysaccharide degradation. Similarly, in this study, we have isolated a bacteria which has algicidal and microcystin degradation property. We observed the mechanism of interaction via; SEM analysis and genome sequencing. Genome sequence data analysis confirms the presence of pathways and genes responsible for algal lysis and conserved region for microcystin degradation.

\section{Materials And Methods}

\subsection{Isolation and cultivation of M. aeruginosa and Rhizobium (AQ_MP) used for interaction studies}

Microcystis aeruginosa was isolated from water samples of Ambazari Lake, Nagpur, Maharashtra. Culture was maintained by subculturing for 30-35 days as defined by (Sangolkar L. N, et al., 2009). M. aeruginosa was poured first with the $\mathrm{OD}_{678} 0.03$, cultured in $250 \mathrm{~mL}$ of conical flasks using BG-11 medium under continuous cool fluorescent light (12:12 light and dark cycle, 3000 lux) at $25 \pm 1^{\circ} \mathrm{C}$ and mechanically shaken a day thrice. Different bacterial cultures were isolated, purified and characterized from the lake water sample published in our previous studies (Pal, M., et al., 2018). Fresh bacterial culture was prepared by inoculation of Rhizobium sp. (AQ_MP) into $250 \mathrm{ml}$ conical flask containing $100 \mathrm{ml}$ of Luria broth media and kept overnight in a shaking incubator $(120 \mathrm{rpm}, 30 \otimes \mathrm{C})$ for 24 hours. Growth curve of Rhizobium sp. (AQ_MP) also analysed (Supplementary Figure 1).

\section{2 aeruginosa cells after exposure to Rhizobium sp. (AQ_MP) : Lysis of algae}

M. aeruginosa $(100 \mathrm{ml})$ suspension were interacted with $10 \mathrm{ml}$ of $1 \mathrm{OD}_{600}$ bacterial suspension (Rhizobium). The experiment was done for ten days, where control and treated samples were collected every day. Samples $(1 \mathrm{ml})$ were centrifuged at $10000 \mathrm{rpm}$ for $10 \mathrm{mins}$ and fixed in $5 \%$ glutaraldehyde, and kept at $4 \llbracket \mathrm{C}$ for Scanning Electron Microscopy (SEM) analysis. Cells (control and experimental) were centrifuged at $10000 \mathrm{rpm}$ and suspended and fixed with $5 \% \mathrm{v} / \mathrm{v}$ glutaraldehyde in $0.1 \mathrm{M}$ phosphate buffer (30 min). Fixed control and experimental cells were centrifuged again, and the supernatant was discarded. Cell pellets were washed three times with $0.1 \mathrm{M}$ phosphate buffer (15 min); dehydration was done with $35 \%$ ethanol (15 min), 50\% ethanol (15 min), 75\% ethanol (15 min), 95\% ethanol (15 min), and two times with $100 \%$ ethanol (15 min). The gold coating of samples was done using Tescan SEM equipment. The cells were then examined in a Vegag3 software, operating at $5.0 \mathrm{kV}$ by a Germany 
scanning electron microscope (Kim, M.,et al., 2020). The total chlorophyll concentration was estimated for the algae-bacteria mixture for ten days by a method described by (Gupta, S. and Pawar, S.B., 2018), wherein filtered dried biomass was extracted with $80 \%$ of ice-chilled acetone for estimation of chlorophyll using wavelength $663.2 \mathrm{~nm}$ and $646.8 \mathrm{~nm}$.

\subsection{Whole-genome sequencing and annotation of Rhizobium (AQ_MP):}

The qualities and quantities of the received bacterial DNA samples were checked by resolved on $1 \%$ Agarose gel followed by quantification using NanoDrop. The gDNA samples were identified on the basis of molecular identification by targeting the bacterial $16 \mathrm{~S}$ gene using Sanger sequencing technique. PCR amplified the fragment of the bacterial 16S gene. A single distinct amplicon band of PCR was observed in the agarose gel. Specific primer was used for DNA sequencing reaction of PCR amplicons. QC passed genomic DNA sample was used for the paired-end sequencing library preparation (after confirmation), using Illumina TruSeqNano DNA Library Prep Kit (Srinivasan, V.B. and Rajamohan, G., 2020, Tikariha, H., et al., 2016). Gene prediction and functional annotation were made using Rapid Annotation using Subsystem Technology (RAST) server (Overbeek, R., et al., 2014). CG Viewer server was also used to create a circular genome that allowed to visualize sequence feature information in the context of sequence analysis outcomes. In this server only, PROKKA annotation was also done to merge CDS, tRNA, tmRNA and rRNA subunits. InterProscan database using pfam system (Patel, D, D et al., 2014) was used to check the presence of the genetic domain of microcystin protein family. Dbcan database was used to check the annotate CAZymes.

\section{Result And Discussion}

\subsection{Interaction study of Microcystis aeruginosa and Rhizobium (AQ_MP):}

In this study, control and experimental Microcystis aeruginosa culture with Rhizobium sp. (AQ_MP) culture was kept for 10 days to check the interaction and degradation of $M$. aeruginosa cells. After the collection of samples, SEM analysis showed the contact between M. aeruginosa and Rhizobium sp. (AQ_MP) cells. In ten days of interaction, $M$. aeruginosa cells were lysed in flask experiments, as demonstrated in our previous studies (Pal M., et al. 2018). Many studies have already been suggested that $M$. aeruginosa have large mucilaginous aggregates comprised of a mucus substance called phycosphere. This mucous region typically comprises associated epiphytic bacteria (Kim, M.,et al., 2019). (Zhang, H., et al., 2011, Gumbo, J.R. and Cloete, T.E., 2011) already published some data on the interaction mechanism between bacteria and $M$. aeruginosa that depicts the $M$. aeruginosa cell membrane's damage, followed by the release of some extracellular substances. These extracellular substances are useful nutrients for bacteria growth. This bacteria-cyanobacteria sometimes shows epiphytic relationships, where the dominant species flourish the most. Total Chlorophyll estimation was also estimated which showed that the chlorophyll level was decreased at the end of the $10^{\text {th }}$ day (Figure 1). 
3.2 Lysis of aeruginosa cells exposed to Rhizobium sp. (AQ_MP) and it's mechanism of action:

SEM was performed to analyze cellular interactions between the Rhizobium sp. (AQ_MP) and $M$. aeruginosa cells. On the first day of experiment, $M$. aeruginosa was observed as a dominant organism. As the incubation continues, the Rhizobium sp. (AQ_MP) cells immersed as in dominant organism indicating towards the lysis of $M$. aeruginosa. Figure 2 shows that from day second onwards, there was a close interaction between the Rhizobium sp. (AQ_MP) and M. aeruginosa cells. SEM image of day $5^{\text {th }}$ and day $7^{\text {th }}$ showed the ruptured $M$. aeruginosa cells. The ruptured cells release the intracellular component, which was utilized as a nutrient by Rhizobium sp. (AQ_MP). Control experiment showed the healthy and dominant $M$. aeruginosa cells after the $10^{\text {th }}$ day. In Figure 3 we hypothesized the actual mechanism of lysis between bacterial and $M$. aeruginosa cells. $M$. aeruginosa cells are surrounded and attached by Rhizobium sp. (AQ_MP) cells, which can destroy the cell wall of the M. aeruginosa, resulting in cell lysis. Our previous study showed that Rhizobium sp. (AQ_MP) cells used microcystin toxin as a carbon source (Pal M., et al., 2018). The present study is an advancement to the previous findings where cell lysis occurred due to enhanced bacterial population. Increased Rhizobium sp. (AQ_MP) cells cause light hindrance, which affects the $M$. aeruginosa cell growth. Rhizobium sp. (AQ_MP) cells also release lytic enzymes, which leads to the destruction of the $M$. aeruginosa cell wall. This destructed $M$. aeruginosa cell becomes a nutrient for the growth of Rhizobium sp. (AQ_MP) cells and continues the deterioration of $M$. aeruginosa cell.

\subsection{Whole-genome sequencing statistics:}

The filtered high-quality PE reads of the bacterial samples mentioned were assembled into scaffolds using SPAdes assembler (v-3.13.0). Nanodrop reading was observed as $152 \mathrm{ng} / \mu \mathrm{l}$. Total data was 616 $\mathrm{Mb}$; the total number of bases were $615,855,661$, total number of reads were observed 2,069,397, total number of scaffolds was 122 . The average scaffold size was (bp) 43,485. Max and min scaffold size was $620,217 \mathrm{bp}$ and $200 \mathrm{bp}$. Whole-genome was submitted to NCBI/Genbank under the accession number JACJVI010000000 as Rhizobium sp. (AQ_MP). After getting the NCBI genome data, proteins/enzymes present in the genome were downloaded from NCBI. FASTA file downloaded from NCBI was then uploaded into the RAST server to check the $M$. aeruginosa lysis and microcystin degradation pathways. CG viewer server database results showed a Circular representation of the Rhizobium sp. (AQ_MP) genome. From outward to inward: ORF (circles 1), CDS (circle 2\&3), GC skew (circle 4), GC content (circle 5), ORF (circle 6), are shown in Figure 4. RAST server data results depict the presence of mlrC gene sequence from 184197 to 185678 in scaffold 2. Size of the sequence was found 1482bp and 494aa (Figure 5). Some mlr (microcystin degrading gene) genes from NCBI were compared with conserved regions of Rhizobium sp. (AQ_MP), and match was observed and checked in pfam and InterProScan (Mitchell, A.L., et al., 2019, Bridge, A.J., et al., 2016). The result of InterProScan for the Rhizobium sp. (AQ_MP) conserved genome sequence is represented in Figure 5. At domain level, our target conserved genome sequence were classified under the protein family IPR009197 and domain IPR015995, IPR010799. This protein family was nearly related to $\mathrm{mlrC}$ domain. This family signifies the $\mathrm{C}$-terminus of a bacterial gene cluster product that is related to the degradation of the toxin microcystin and is encoded 
in the $\mathrm{m} / \mathrm{rgene}$ cluster. Phylogenetic tree of some mlrC sequence (NCBI) was compared with conserved sequences of Rhizobium sp. (AQ_MP) (Figure 6). Glycosyltransferase gene was also compared as outer protein family. It was seen that Rhizobium sp. (AQ_MP) conserved region was related to many Sphingopyxis sp. and Sphingomonas mlrC genes.

\subsection{Microcystin degrading pathway:}

Analysis of the whole genome shotgun sequencing data depicts that Rhizobium sp. (AQ_MP) followed a Glutathione metabolic pathway (Sies, H., et al., 1980) for the degradation of microcystins, in which glutathione-S-transferase (gst) (MBC2773493.1) and gamma-glutamyltransferase (tgm) (MBC2775265.1) enzyme was present. Microcystin has ADDA and Mdha site in which in the Mdha site, glutathione was attached and formed Microcystin-RR-glutathione (J.R., et al., 2014). Due to the presence of Gamma-glutamyltransferase enzyme Microcystin-RR-glutathione, get cleaved, and gamma glutamic acid was released, which leads to the formation of Microcystin-RR-cysteine-Glycine (Wang, X., et al., 2018; Lance, E., et al., 2014). Cyc-gly Dipeptidase (dug) was used to cleave the gamma-glutamylcysteine intermediate's glycineto get the cysteine-conjugated product, as a result, oxidized by acetyl transferaseacetyl co-A (acat) to form the mercapturic acid metabolite (Manahan, S.E. 2003), which is Microcystin-RRMercapturic acid (Figure 7). Another pathway was found for the degradation of microcystin, where in Rhizobium sp. (AQ_MP), three enzymes jointly denoted as microcystinase operate in a sequential pathway to degrade MC. The first enzyme Microcystinase $\mathrm{C}$ precursor $(\mathrm{mlrC})$ linearizes microcystin through the cleavage of the peptide ring at the ADDA-arginine bond. The second enzyme, linearized microcystinase ( $\mathrm{mlrB}$ ) cleaves this linear intermediate at the alanine-leucine bond, yielding a peptide intermediate of ADDA-Glu-Mdha-Ala (Goldberg, J., et al., 1995). The final enzyme degrades the products formed by the first two enzymes and releases ADDA from the tetrapeptide intermediate (Figure 4). Genes denoting linearized microcystinase $(\mathrm{mlrB})$ was the conserved region of beta-lactamase (scaffold 22 , sequence $=33495-35177$ ), was annotated via RAST, InterProscan, and checked via Uniprot identity (supplementary figure 2).

\subsection{Polysaccharides degradation enzymes:}

CAZymes are essential enzymes for polysaccharide degradation (Srivastava, S., et al., 2020). dbCAN meta server data showed the presence of many CAZyme in the Rhizobium sp. (AQ_MP) genome like 147 Glycoside hydrolases (GH), 140 Glycosyltransferases (GT), 15 carboxylesterase (CE), 13 carbohydratebinding modules, and four polysaccharide lyases (PL). Table 1 is showing different CAZymes present in the Rhizobium sp. (AQ_MP) genome. It shows a distinct specificity for polysaccharides (Xing, P., et al., 2015), glycoproteins, and proteins degradation, which is required for the growth of cyanobacteria. For the degradation of the algal polysaccharides, PLs and GHs are anticipated, including chitin, agarose, fucoside (N-linked glycan), fucoidan (fucose-containing sulfated polysaccharides), rhamnogalacturonan, homogalacturonan, starch (glucan), and xylan. Among these, in red seaweed, agarose is a unique cell wall polysaccharide; rhamnogalacturonan and homogalacturonan are the pectic compounds generally present in the seaweed cell walls; likewise xylan is present in the green algae, red algae, and plant cell 
walls. The presence of these CAZymes in the Rhizobium sp. (AQ_MP) suggests the better degradation potential of cyanobacterial polysaccharides. PLO and PL 5, alginate lyases indicated that bacteria could cut down alginate into different oligosaccharides or monosaccharides (Zhu, Y., et al., 2016). The annotated agarase (GH16) and galactosidase (GH2) likely degrade agarose (Hehemann, J.H., et al., 2010). Endo-1,4-glucanase (GH5) and Glucosidase (GH32) are cellulose degraders (Taylor, L.E., et al., 2006). Chitin can be hydrolyzed by Rhizobium sp. (AQ_MP) with hexosaminidase (GH19) and chitinase (GH16). The annotated L-fucosidases (GH65 and GH33) can degrade fucoidans and fucosides (Ale, M.T., et al., 2011). Homogalacturonan could be degraded by polygalacturonase (GH28). Two bi-functional enzymes, i.e. xylosidase/ L-arabinofuranosidase (GH3), are expected to degrade arabinoxylan hemicelluloses (Lee, R.C., et al., 2003). The unsaturated rhamnogalacturonyl hydrolase (GH105), Larabinofuranosidase (GH3), and L-rhamnosidase (GH78) can be degrade rhamnogalacturonan (Weiner, R.M., et al., 2008). Starch can be hydrolyzed with glucosidases (GH36) and amylase (GH13). The putative xylosidase (GH38 and GH43), endo-1,4-xylanase (GH43), and L-arabinofuranosidase (GH3) can degrade xylan. 540 transporters were also found, of which 353 were ABC transporter. Transporter proteins are also responsible for the degradation of microcystins. PhzF family phenazine biosynthesis protein (MBC2772314.1) was also found in the genome related to algal biodegradation (Dakhama, A., et al., 1993). These loci play a pivotal role to degrade algal polysaccharides and microcystins. In most bacterial genomes, CAZymes typically account for $2 \%$ of the genes and occasionally exceed $5 \%$ for those bacteria which are specifically responsible for carbohydrate degradation (Bohra, V., et al., 2019). This requires the niche-adapted microbial experts for the rigorous breakdown of composite polysaccharide mixtures present in nature. In bacterial systems, polysaccharide degradation genes are organized in larger regulon and operon structures, termed as polysaccharide utilization loci (PULs). PULs encode many transcriptional regulators, sulphatases, transporters, and carbohydrate-active enzymes (CAZymes). CAZymes are enzymes for deterioration, and synthesis/modification, polysaccharide binding/recognition in glycoside hydrolases (GHs), carbohydrate-binding modules (CBMs), carbohydrate esterases (CEs), polysaccharide lyases (PLs), and glycosyltransferases (GTs) (Srivastava, S., et al., 2020). Above mentioned description suggests that these loci play a crucial role in Bacteroidetes polysaccharide biodeterioration. In the Rhizobium sp. (AQ_MP), it exposed the ability to reducea wide range of cyanobacterial polysaccharides.

RAST server data exhibited the presence of siderophores in the Rhizobium sp. (AQ_MP) genome. Iron is a crucial element essential for key biotic processes. The common bacterial groups necessitate iron for existence and progression. Bioavailability of Iron is limited which is a persistent source of pressure in many biological structures. There is accruing indication that Fe restricts phytoplankton biomass in the equatorial Pacific (Wells, M.L., et al., 1994), the North Pacific gyre (Martin et al., 1989; Liu, Z.Z., et al., 2014), and the Southern Ocean (Helbling et al., 1991). For microorganisms obtaining iron is a significant challenge; capturing and integrating iron governs their existence. Cyanobacteria and algae, particularly responsible for biomass's primary production, requiresten times higher iron content than nonphotosynthetic prokaryotes (Brand, 1991). Bacteria, fungi, microalgae, and many higher plants have established specific approaches for low iron bioavailability. Siderophores secretion is one of the 
approaches among them. Siderophores are the molecules that chelate iron with high affinity (Guerinor, 1994). Siderophores extracellularly solubilizes the iron from minerals of organic substances and transport them into cells when there is deprivation of iron. Photosynthesis and capturing light energy are closely related with photosynthetic pigments. In photosynthesis, iron plays a crucial role in chlorophyll-a production (Imai et al., 1999). Studies say cyanobacteria requires higher iron uptake that other algae (Brand, 1991). Liu, Z.Z., et al., 2014 has suggested that due to the presence of siderophores, photosynthetic pigment synthesis was inhibited in M. aeruginosa. In our study, Rhizobium sp. (AQ_MP) is connected to siderophore release, which indicates the inhibition of photosynthetic pigment synthesis. It could be responsible for the inhibition of $M$. aeruginosa growth due to the low bioavailability of iron (Martin, J.H., et al., 1989). In previous studies, it was observed that bacteria and cyanobacteria tend to compete in freshwater for the low bioavailability of iron (Liu, Z.Z., et al., 2014). Protein FASTA sequences of these loci were downloaded from NCBI and were submitted to Phyre software, and Pymol generates the structures of different siderophore and CAZymes (Supplementary Figure 3).

Nitrogen is a good source for the growth of cyanobacteria. Rhizobium sp. (AQ_MP) exhibited denitrification activity against $M$. aeruginosa. The denitrification pathway was present in the RAST server (Supplementary Figure 4). nar, nir, nos and nor are the genes responsible for the conversion of nitrate to ammonia (Tikariha, H. and Purohit, H.J., 2019) were also present in the genome. Some previously published work has been shown how significant denitrification is to control algal blooms (Jiang, X., et al., 2020).

\section{Conclusion}

Rhizobium sp. (AQ_MP) was isolated from the lake water sample, which showed the lysis of harmful Cyanobacterial sp. Microcystis aeruginosa. Scanning electron microscopy (SEM) and chlorophyll estimation revealed the algicidal property of Rhizobium sp. (AQ_MP). Genome analysis revealed that Rhizobium sp. (AQ_MP) possess secretion ability for extracellular substances like CAZymes responsible for algal polysaccharide degradation and utilizes the nutrient released from lysed cyanobacterial cells. The presence of toxin (microcystin) degradative pathways and gene clusters for polysaccharide degradation confirmed toxin degradation as well as algicidal characteristics in Rhizobium sp. (AQ_MP).

\section{Declarations}

\section{Acknowledgment:}

Authors are grateful to Director, CSIR-NEERI (KRC No.: CSIR-NEERI/KRC/2020/NOV/EBGD/4), for providing all the necessary infrastructure required for the experimentation. The authors also thank DBT (BT/PR16149/NER/95/85/2015 dated 19/01/2017) for the funding.

6. Conflict of interest: 
All authors have mutually agreed to submit this manuscript to this Journal. All the authors declare that they have no conflict of interest.

\section{References}

Ale, M.T., Mikkelsen, J.D. and Meyer, A.S., 2011. Important determinants for fucoidan bioactivity: A critical review of structure-function relations and extraction methods for fucose-containing sulfated polysaccharides from brown seaweeds. Marine drugs, 9(10), pp.2106-2130.

Bohra, V., Dafale, N.A. and Purohit, H.J., 2019. Understanding the alteration in rumen microbiome and CAZymes profile with diet and host through comparative metagenomic approach. Archives of Microbiology, 201(10), pp.1385-1397.

Bridge, A.J., Babbitt, P.C., Smithers, B., Huang, X., Huang, H., Lu, S., Mi, H., Marchler-Bauer, A., Thomas, P.D., Richardson, L. and Rawlings, N.D., 2016. InterPro in 2017--beyond protein family and domain annotations.

Carmichael, W.W., 2001. Health effects of toxin-producing cyanobacteria:"The CyanoHABs". Human and ecological risk assessment: An International Journal, 7(5), pp.1393-1407.

Corbel, S., Mougin, C. and Bouaïcha, N., 2014. Cyanobacterial toxins: modes of actions, fate in aquatic and soil ecosystems, phytotoxicity and bioaccumulation in agricultural crops. Chemosphere, 96, pp.1-15.

Dakhama, A., De la Noüe, J. and Lavoie, M.C., 1993. Isolation and identification of antialgal substances produced byPseudomonas aeruginosa. Journal of Applied Phycology, 5(3), pp.297-306.

Goldberg, J., Huang, H.B., Kwon, Y.G., Greengard, P., Nairn, A.C. and Kuriyan, J., 1995. Three-dimensional structure of the catalytic subunit of protein serine/threonine phosphatase-1. Nature, 376(6543), pp.745753.

Gumbo, J.R. and Cloete, T.E., 2011. The mechanism of Microcystis aeruginosa death upon exposure to Bacillus mycoides. Physics and Chemistry of the Earth, Parts $A / B / C, 36(14-15)$, pp.881-886.

Gupta, S. and Pawar, S.B., 2018. Mixotrophic cultivation of microalgae to enhance the quality of lipid for biodiesel application: effects of scale of cultivation and light spectrum on reduction of a-linolenic acid. Bioprocess and biosystems engineering, 41(4), pp.531-542.

Hehemann, J.H., Correc, G., Barbeyron, T., Helbert, W., Czjzek, M. and Michel, G., 2010. Transfer of carbohydrate-active enzymes from marine bacteria to Japanese gut microbiota. Nature, 464(7290), pp.908-912.

Jiang, X., Gao, G., Zhang, L., Tang, X., Shao, K., Hu, Y. and Cai, J., 2020. Role of algal accumulations on the partitioning between $\mathrm{N} 2$ production and dissimilatory nitrate reduction to ammonium in eutrophic lakes. Water Research, 183, p.116075. 
Kim, M., Shin, B., Lee, J., Park, H.Y. and Park, W., 2019. Culture-independent and culture-dependent analyses of the bacterial community in the phycosphere of cyanobloom-forming Microcystis aeruginosa. Scientific reports, 9(1), pp.1-13.

Lampert, W., 1987. Laboratory studies on zooplankton-cyanobacteria interactions. New Zealand journal of marine and freshwater research, 21(3), pp.483-490.

Lance, E., Petit, A., Sanchez, W., Paty, C., Gerard, C. and Bormans, M., 2014. Evidence of trophic transfer of microcystins from the gastropod Lymnaea stagnalis to the fish Gasterosteus aculeatus. Harmful Algae, 31, pp.9-17.

Lee, R.C., Hrmova, M., Burton, R.A., Lahnstein, J. and Fincher, G.B., 2003. Bifunctional Family 3 Glycoside Hydrolases from Barley with a-l-Arabinofuranosidase and $\beta$-d-Xylosidase Activity characterization, primary structures, and cooh-terminal processing. Journal of Biological Chemistry, 278(7), pp.5377-5387.

Liu, Z.Z., Zhu, J.P., Li, M., Xue, Q.Q., Zeng, Y. and Wang, Z.P., 2014. Effects of freshwater bacterial siderophore on Microcystis and Anabaena. Biological Control, 78, pp.42-48.

Manahan, S.E. Toxicological Chemistry and Biochemistry, 3rd ed.; CRC Press: Boca Raton, FL, USA, 2003; pp.142-153

Mann, A.J., Hahnke, R.L., Huang, S., Werner, J., Xing, P., Barbeyron, T., Huettel, B., Stüber, K., Reinhardt, R., Harder, J. and Glöckner, F.O., 2013. The genome of the alga-associated marine flavobacterium Formosa agariphila KMM $3901 \mathrm{~T}$ reveals a broad potential for degradation of algal polysaccharides. Applied and environmental microbiology, 79(21), pp.6813-6822.

Martin, J.H., Gordon, R.M., Fitzwater, S. and Broenkow, W.W., 1989. VERTEX: phytoplankton/iron studies in the Gulf of Alaska. Deep Sea Research Part A. Oceanographic Research Papers, 36(5), pp.649-680.

Mitchell, A.L., Attwood, T.K., Babbitt, P.C., Blum, M., Bork, P., Bridge, A., Brown, S.D., Chang, H.Y., El-Gebali, S., Fraser, M.I. and Gough, J., 2019. InterPro in 2019: improving coverage, classification and access to protein sequence annotations. Nucleic acids research, 47(D1), pp.D351-D360.

Overbeek, R., Olson, R., Pusch, G.D., Olsen, G.J., Davis, J.J., Disz, T., Edwards, R.A., Gerdes, S., Parrello, B., Shukla, M. and Vonstein, V., 2014. The SEED and the Rapid Annotation of microbial genomes using Subsystems Technology (RAST). Nucleic acids research, 42(D1), pp.D206-D214.

Paerl, H.W., Hall, N.S. and Calandrino, E.S., 2011. Controlling harmful cyanobacterial blooms in a world experiencing anthropogenic and climatic-induced change. Science of the total environment, 409(10), pp.1739-1745.

Pal, M., Pal, S., Qureshi, A. and Sangolkar, L.N., 2018. Perspective of cyanobacterial harmful algal bloom (HAB) mitigation: Microcystis toxin degradation by bacterial consortia. Indian Journal of Experimental Biology. 
Pal, M., Yesankar, P.J., Dwivedi, A. and Qureshi, A., 2020. Biotic control of harmful algal blooms (HABs): A brief review. Journal of Environmental Management, 268, p.110687.

Pearson, L., Mihali, T., Moffitt, M., Kellmann, R. and Neilan, B., 2010. On the chemistry, toxicology and genetics of the cyanobacterial toxins, microcystin, nodularin, saxitoxin and cylindrospermopsin. Marine drugs, 8(5), pp.1650-1680.

Rinehart, K.L., Namikoshi, M. and Choi, B.W., 1994. Structure and biosynthesis of toxins from blue-green algae (cyanobacteria). Journal of applied phycology, 6(2), pp.159-176.

Sangolkar, L.N., Maske, S.S., Muthal, P.L., Kashyap, S.M. and Chakrabarti, T., 2009. Isolation and characterization of microcystin producing Microcystis from a Central Indian water bloom. Harmful algae, 8(5), pp.674-684.

Schmidt, J.R., Wilhelm, S.W. and Boyer, G.L., 2014. The fate of microcystins in the environment and challenges for monitoring. Toxins, 6(12), pp.3354-3387.

Sies, H., Wahlländer, A., Waydhas, C., Soboll, S. and Häberle, D., 1980. Functions of intracellular glutathione in hepatic hydroperoxide and drug metabolism and the role of extracellular glutathione. Advances in enzyme regulation, 18, pp.303-320.

Srinivasan, V.B. and Rajamohan, G., 2020. Comparative genome analysis and characterization of a MDR Klebsiella variicola. Genomics.

Srivastava, S., Dafale, N.A. and Purohit, H.J., 2020. Functional genomics assessment of lytic polysaccharide mono-oxygenase with glycoside hydrolases in Paenibacillus dendritiformis CRN18. International Journal of Biological Macromolecules, 164, pp.3729-3738.

Taylor, L.E., Henrissat, B., Coutinho, P.M., Ekborg, N.A., Hutcheson, S.W. and Weiner, R.M., 2006. Complete cellulase system in the marine bacterium Saccharophagus degradans strain 2-40T. Journal of Bacteriology, 188(11), pp.3849-3861.

Tikariha, H., and Purohit, H.J., 2019. Assembling a genome for novel nitrogen-fixing bacteria with capabilities for utilization of aromatic hydrocarbons. Genomics, 111(6), pp.1824-1830.

Tikariha, H., Pal, R.R., Qureshi, A., Kapley, A. and Purohit, H.J., 2016. In silico analysis for prediction of degradative capacity of Pseudomonas putida SF1. Gene, 591(2), pp.382-392.

Wang, X., Wu, J., Jian, S., Yang, G., Hu, B. and Wen, C., 2018. Microcystin induction small Maf protein involve in transcriptional regulation of GST from freshwater mussel Cristaria plicata. Gene, 660, pp.51-61.

Weiner, R.M., Taylor II, L.E., Henrissat, B., Hauser, L., Land, M., Coutinho, P.M., Rancurel, C., Saunders, E.H., Longmire, A.G., Zhang, H. and Bayer, E.A., 2008. Complete genome sequence of the complex 
carbohydrate-degrading marine bacterium, Saccharophagus degradans strain 2-40 T. PLoS Genet, 4(5), p.e1000087.

Wells, M.L., Price, N.M. and Bruland, K.W., 1994. Iron limitation and the cyanobacterium Synechococcus in equatorial Pacific waters. Limnology and oceanography, 39(6), pp.1481-1486.

Xing, P., Hahnke, R.L., Unfried, F., Markert, S., Huang, S., Barbeyron, T., Harder, J., Becher, D., Schweder, T., Glöckner, F.O. and Amann, R.I., 2015. Niches of two polysaccharide-degrading Polaribacter isolates from the North Sea during a spring diatom bloom. The ISME journal, 9(6), pp.1410-1422.

Zhang, H., Yu, Z., Huang, Q., Xiao, X., Wang, X., Zhang, F., Wang, X., Liu, Y. and Hu, C., 2011. Isolation, identification and characterization of phytoplankton-lytic bacterium $\mathrm{CH}-22$ against Microcystis aeruginosa. Limnologica, 41(1), pp.70-77.

Zhang, J., Lu, Q., Ding, Q., Yin, L. and Pu, Y., 2017. A novel and native microcystin-degrading bacterium of Sphingopyxis sp. isolated from lake Taihu. International journal of environmental research and public health, 14(10), p.1187.

Zhu, Y., Chen, P., Bao, Y., Men, Y., Zeng, Y., Yang, J., Sun, J. and Sun, Y., 2016. Complete genome sequence and transcriptomic analysis of a novel marine strain Bacillus weihaiensis reveals the mechanism of brown algae degradation. Scientific reports, 6(1), pp.1-10.

\section{Table}

Table 1: CAZymes annotated in Rhizobium (AQ_MP) genome 


\begin{tabular}{|c|c|c|}
\hline AAO & 1 & $A B C$ transporter \\
\hline AA1 & 1 & $\mathrm{ABC}$ transporter \\
\hline AA2 & 1 & $\mathrm{ABC}$ transporter \\
\hline AA3 & 19 & cellobbiose dehydrogenase \\
\hline AA4 & 1 & vanillyl- alcohol oxidase \\
\hline AA6 & 2 & 1,4-benzoquinone reductase \\
\hline AA7 & 2 & $A B C$ transporter \\
\hline CBM13 & 1 & Carbohydrate binding modules \\
\hline CBM32+CBM56 & 1 & Polygalactouronic acid \\
\hline CBM5 & 1 & Carbohydrate binding modules \\
\hline CBM50 & 10 & Carbohydrate binding modules \\
\hline CE1 & 3 & Carboxylesterase \\
\hline CE11 & 4 & Carboxylesterase \\
\hline CE12 & 1 & Carbohydrate esterase \\
\hline CE4 & 4 & Carboxylesterase \\
\hline CE9 & 3 & Carbohydrate esterase \\
\hline $\mathrm{GHO}$ & 6 & Glycoside hydrolase \\
\hline $\mathrm{GH} 1$ & 3 & -Glucosidase, -galactosidase, -mannosidase \\
\hline GH101 & 1 & Glycoside hydrolase \\
\hline GH102 & 3 & Glycoside hydrolase \\
\hline GH103 & 12 & Glycoside hydrolase \\
\hline GH105 & 3 & Glycoside hydrolase \\
\hline $\mathrm{GH} 13$ & 26 & a- amylase \\
\hline $\mathrm{GH} 16$ & 4 & Endo- -1,3-glucanase \\
\hline $\mathrm{GH} 19$ & 4 & Chitinase \\
\hline $\mathrm{GH} 2$ & 7 & $\beta$-galactosidase \\
\hline $\mathrm{GH} 20$ & 3 & Glycoside hydrolase \\
\hline $\mathrm{GH} 23$ & 14 & Glycoside hydrolase \\
\hline GH25 & 6 & Glycoside hydrolase \\
\hline
\end{tabular}




\begin{tabular}{|c|c|c|}
\hline $\mathrm{GH} 28$ & 4 & a -1,4-Galacturonanase (polygalacturonase), Exo-polygalacturonase \\
\hline $\mathrm{GH} 3$ & 6 & $\begin{array}{l}-1,4-G l u c o s i d a s e,-1,4-x y l o s i d a s e,-1,3-g l u c o s i d a s e, \text { alpha-L- } \\
\text { arabinofuranosidase }\end{array}$ \\
\hline $\mathrm{GH} 32$ & 3 & Glycoside hydrolase \\
\hline $\mathrm{GH} 33$ & 6 & Glycoside hydrolase \\
\hline $\mathrm{GH} 36$ & 4 & a galactosidase \\
\hline GH38 & 3 & Glycoside hydrolase \\
\hline $\mathrm{GH} 42$ & 6 & Glycoside hydrolase \\
\hline $\mathrm{GH} 43$ & 3 & -1,4-Xylosidase, -arabinofuranosidase, -endoarabinanase, -1,4-endoxylanase \\
\hline GH5 & 3 & -1,4-Endoglucanase, -1,4-endoxylanase, -1,4-endomannanase, licheninase \\
\hline GH51 & 3 & Cellulase \\
\hline GH6 & 1 & Nonreducing end cellobiohydrolase, -1,4-endoglucanase \\
\hline GH65 & 1 & Glycoside hydrolase \\
\hline GH78+CBM67 & 6 & Glycoside hydrolase \\
\hline GH8 & 3 & Polygalacturonases \\
\hline GH94+GT84 & 3 & $\begin{array}{l}\text { Cellobiose phosphorylase, cellodextrin phosphorylase, chitobiose } \\
\text { phosphorylase }\end{array}$ \\
\hline GT0 & 3 & Glycosyltransferases \\
\hline GT13 & 1 & Glycosyltransferases \\
\hline GT19 & 3 & Glycosyltransferases \\
\hline GT2 & 34 & Glycosyltransferases \\
\hline GT21 & 3 & Glycosyltransferases \\
\hline GT25 & 7 & Glycosyltransferases \\
\hline GT26 & 7 & Glycosyltransferases \\
\hline GT28 & 3 & Glycosyltransferases \\
\hline GT30 & 3 & Glycosyltransferases \\
\hline GT35 & 3 & Glycosyltransferases \\
\hline GT4 & 39 & Glycosyltransferases \\
\hline GT41 & 3 & Glycosyltransferases \\
\hline GT5 & 7 & Glycosyltransferases \\
\hline
\end{tabular}




\begin{tabular}{|lll|} 
GT51 & 15 & Glycosyltransferases \\
GT81 & 6 & Glycosyltransferases \\
GT83 & 2 & Glycosyltransferases \\
GT90 & 1 & Glycosyltransferases \\
PL0 & 1 & Polysaccharide lyases \\
\hline PL5 & 3 & Polysaccharide lyases \\
\hline
\end{tabular}

\section{Figures}

\section{Total Chlorophyll}

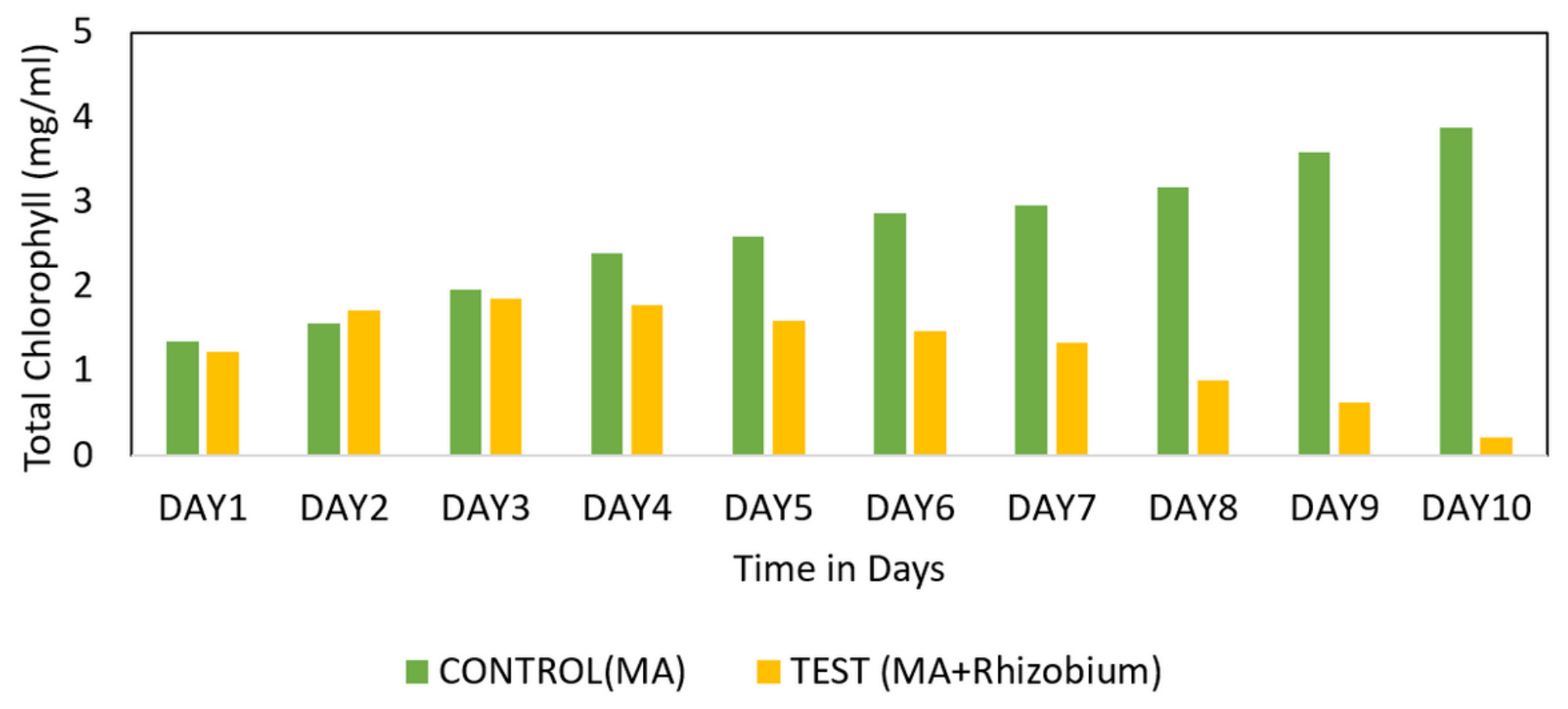

Figure 1

Total chlorophyll estimated; Rhizobium-Microcystis aeruginosa (MA) interaction by acetone method. 


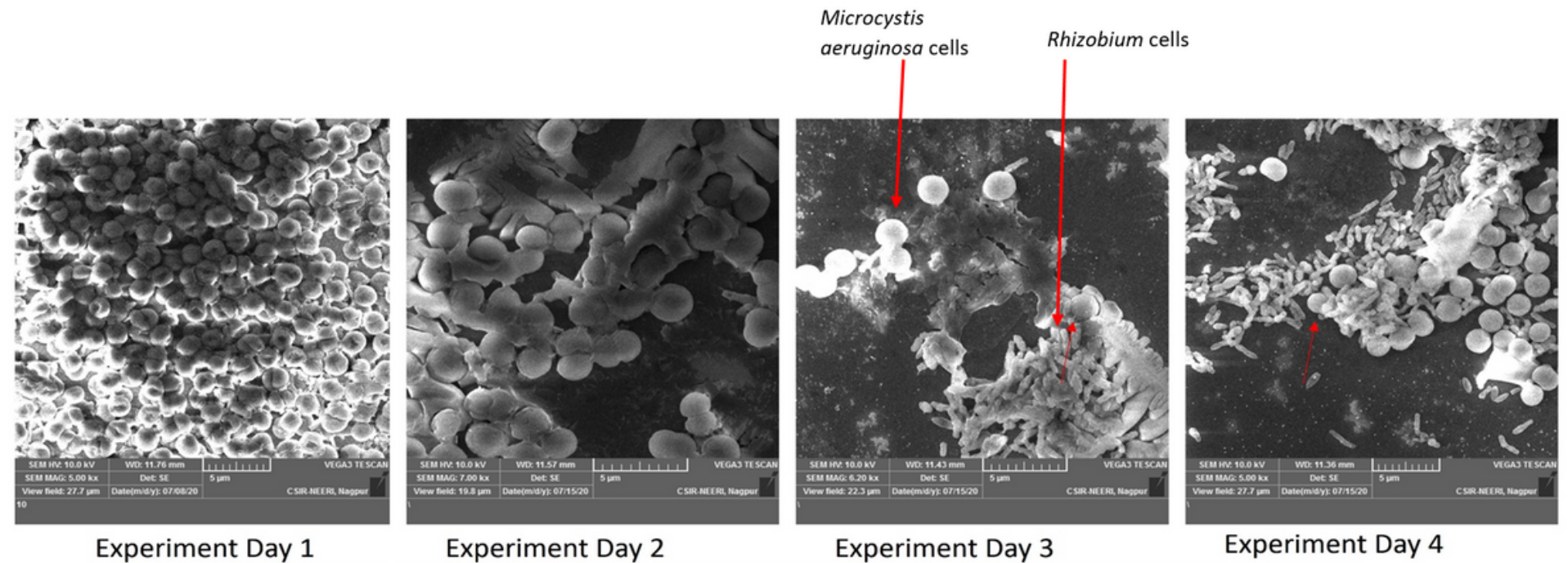

Experiment Day 1

Experiment Day 2

Experiment Day 3

Experiment Day 4

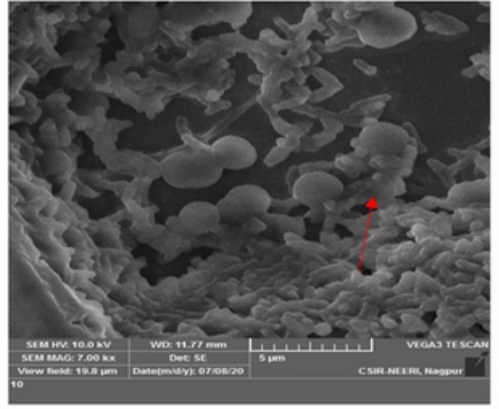

Experiment Day 5

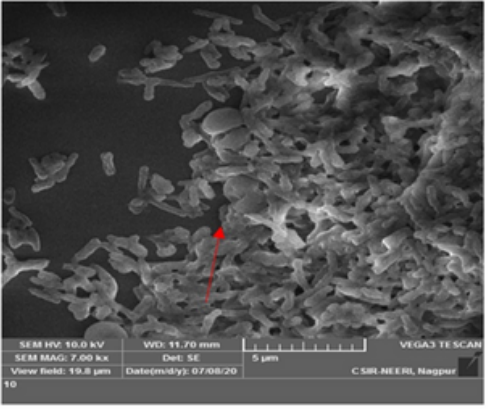

Experiment Day 7

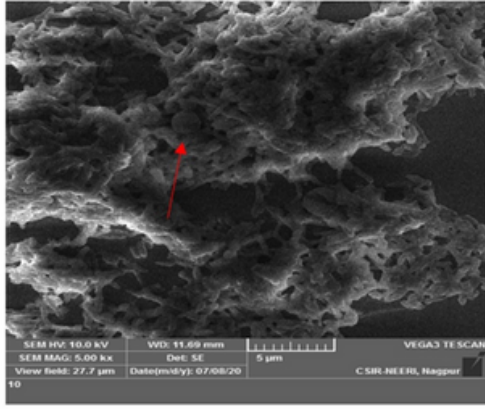

Experiment Day 10

\section{Figure 2}

Scanning electron microphotographs of M. aeruginosa treated with Rhizobium bacterial culture Day1 Day 10 observation

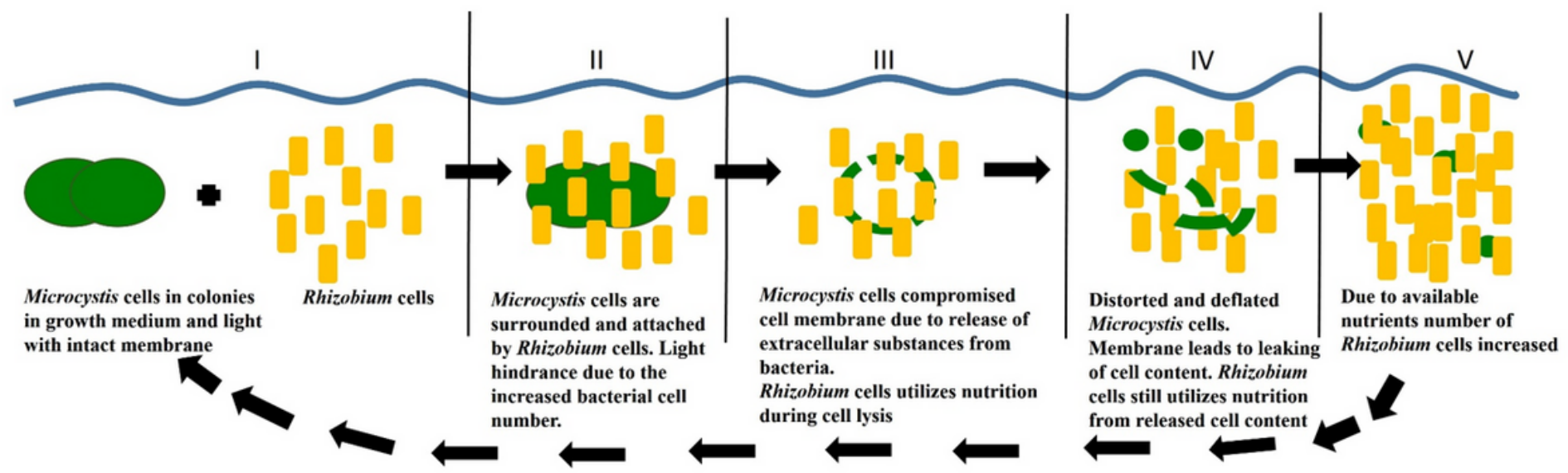

\section{Figure 3}

Mechanism of lysis of Microcystis during interaction with Rhizobium cells, microcystis cells compromised cell membranes due to the release of extracellular substances from bacteria. 

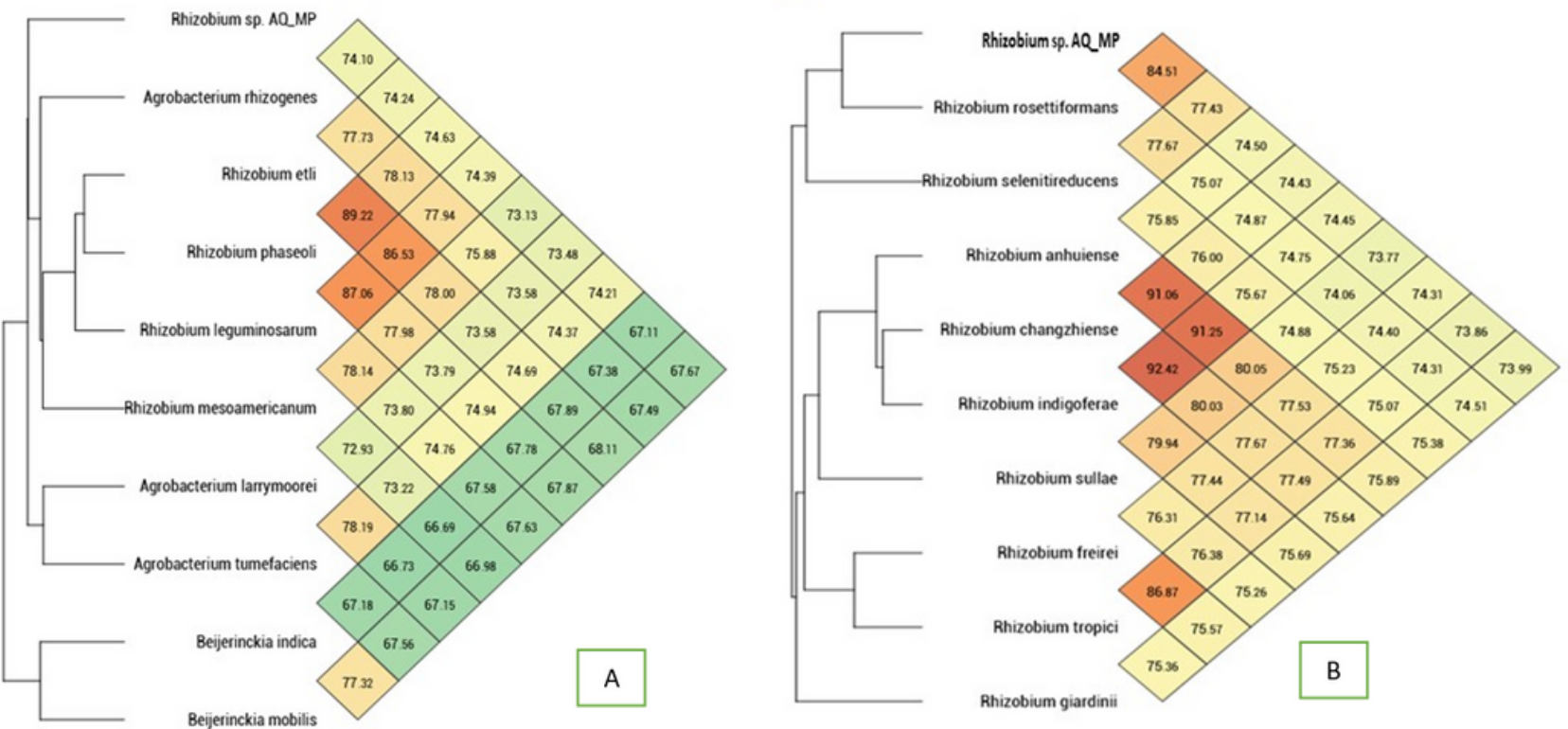

\section{Figure 4}

Heatmap generated with OrthiANI among closely related species of Rhizobium sp. (A), where Rhizobium rosettiformas is showing $84.51 \%$ similarity with our genome (B). 


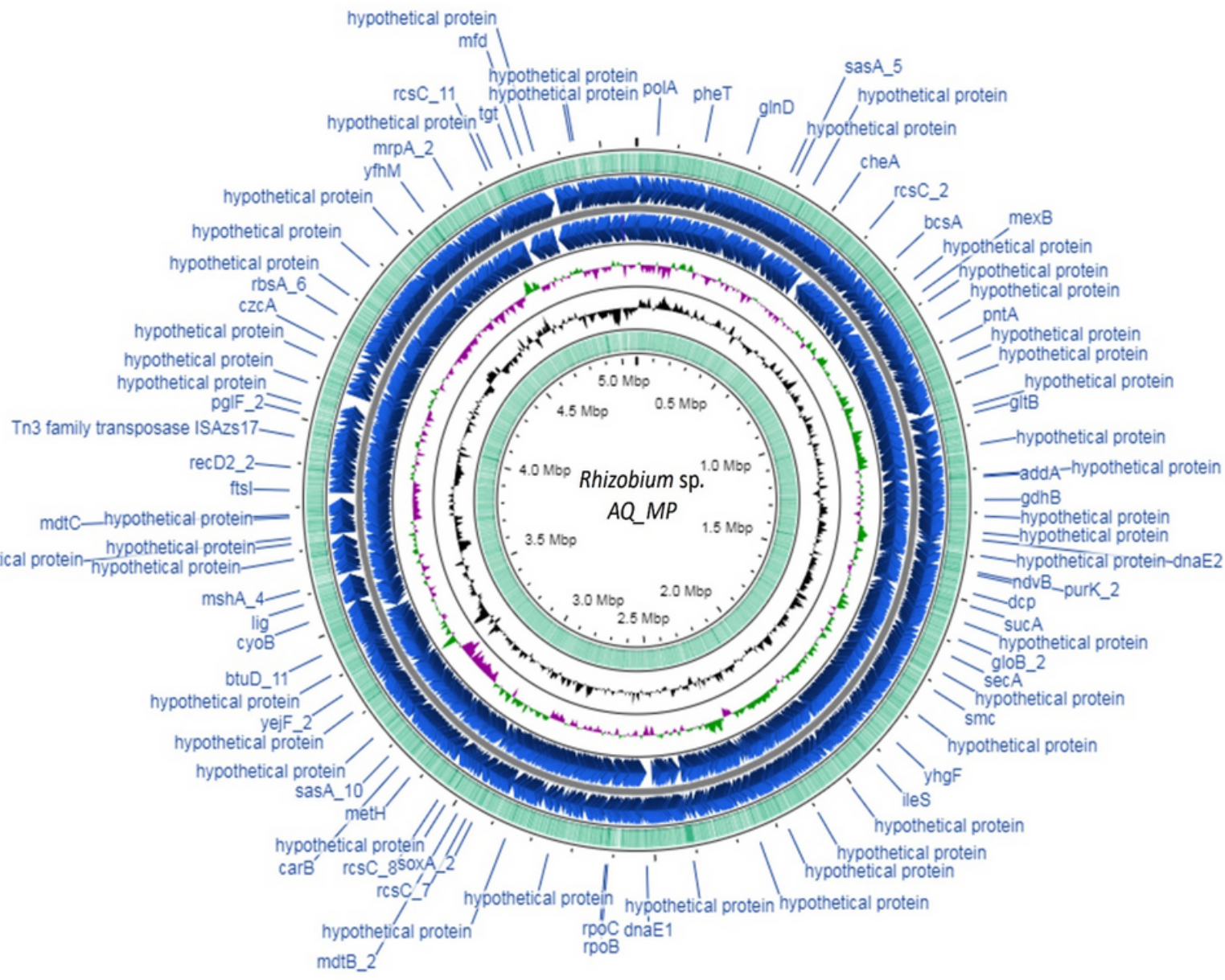

\section{Figure 5}

Circular representation of the Rhizobium genome. From outward to inward: ORF (circles 1), CDS (circle 2\&3), GC skew (circle 4), GC content (circle 5), ORF (circle 6), are shown. Genome picture is retrieved from CGView server using default parameters 


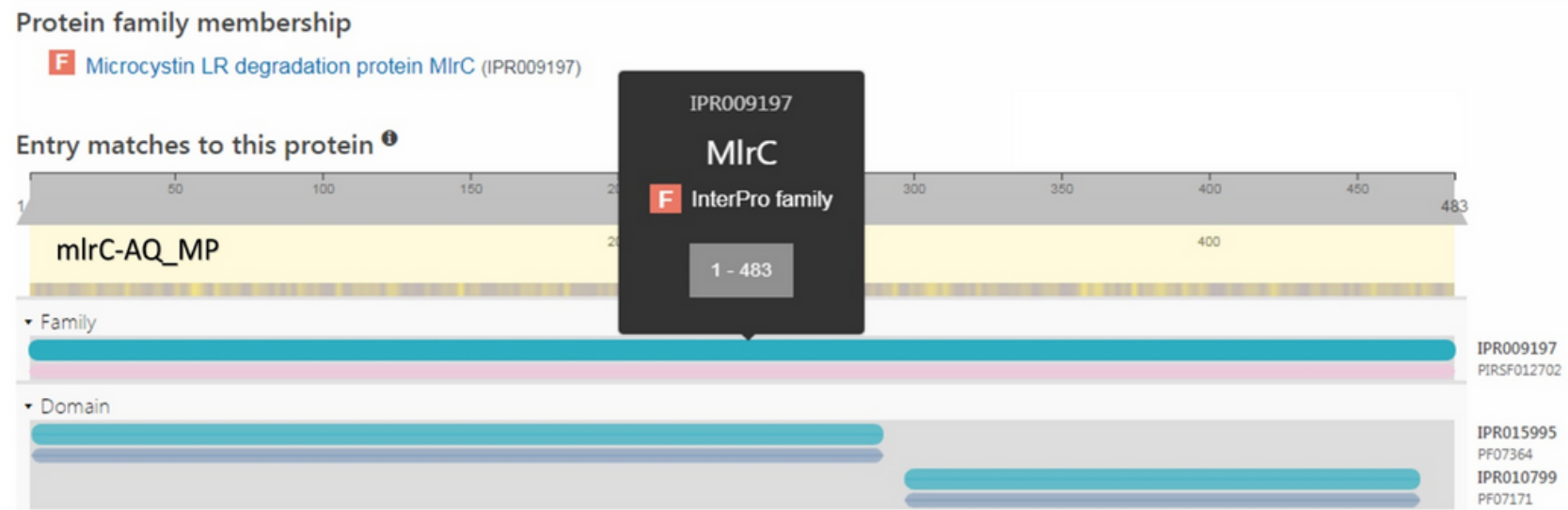

R. $s p$. AQ_MP
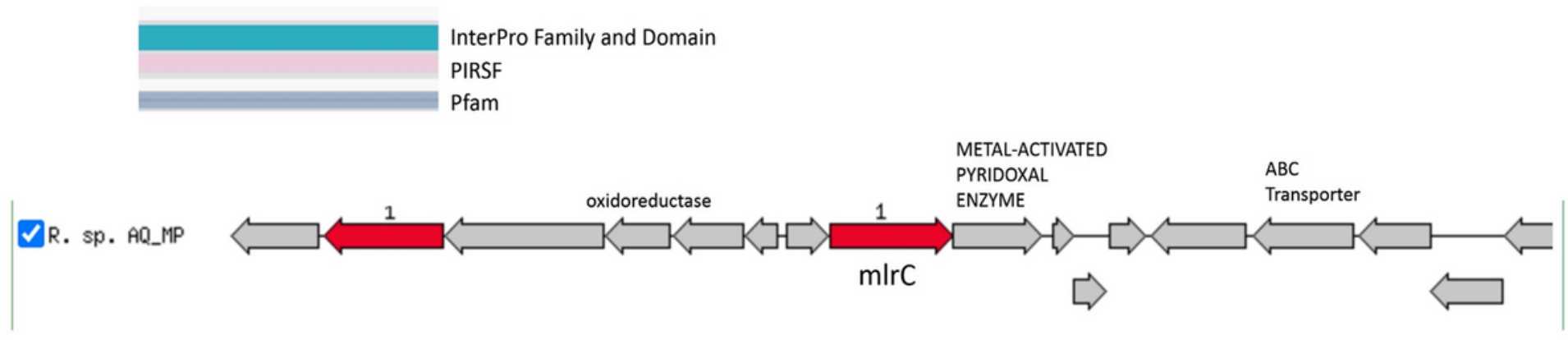

\section{Figure 6}

Gene structure of annotated mlrC gene in Rhizobium. Protein structure of annotated mlrC conserved sequence was scanned through InterProScan tool to identify the multimodular domains of mlrC. 


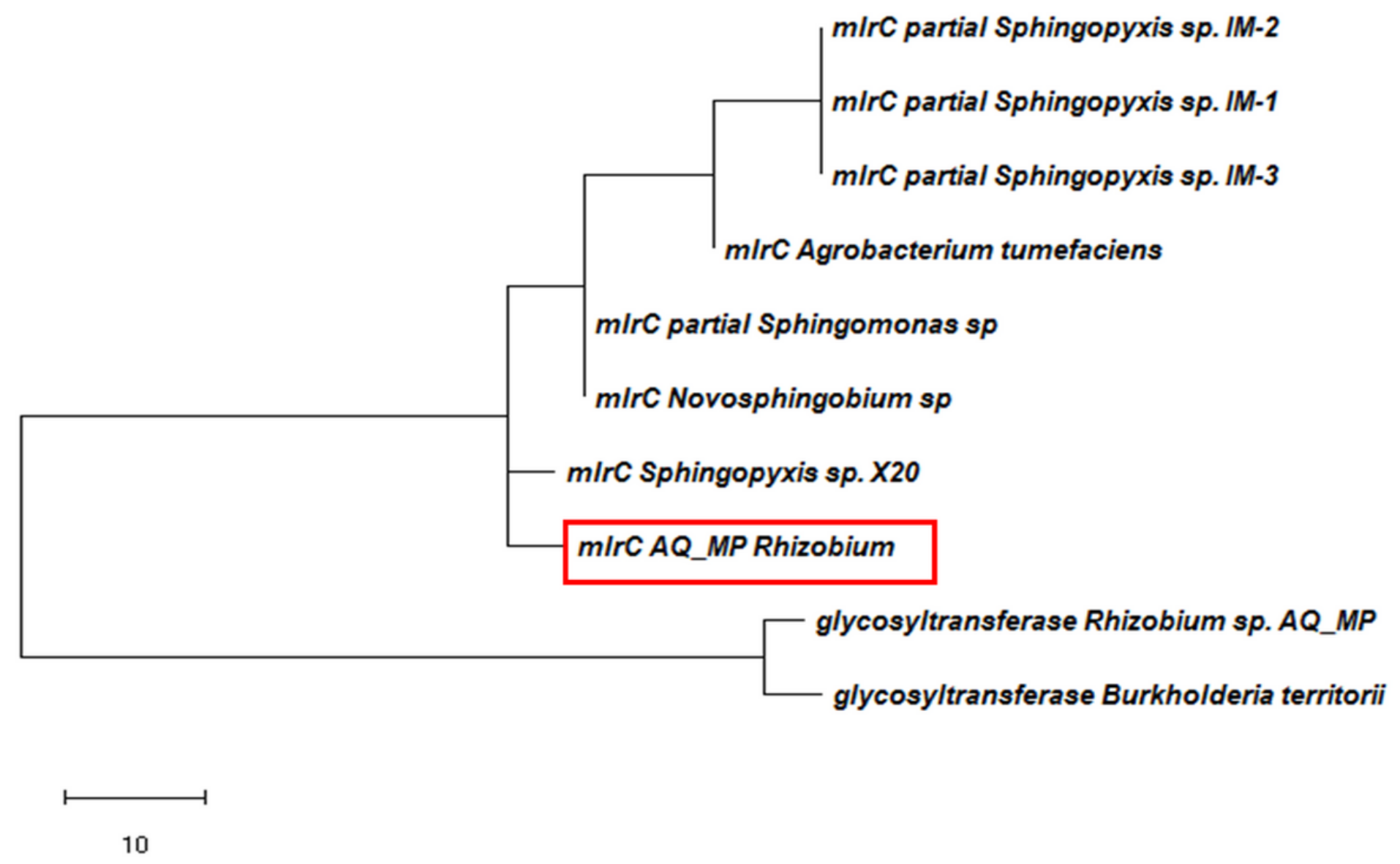

Figure 7

Phylogenetic tree construction of different bacterial mlrC gene with Rhizobium AQ_MP conserved region. AQ_MP sequences are BLAST results of WGS of Rhizobium with different other bacterial mlrC protein sequences 


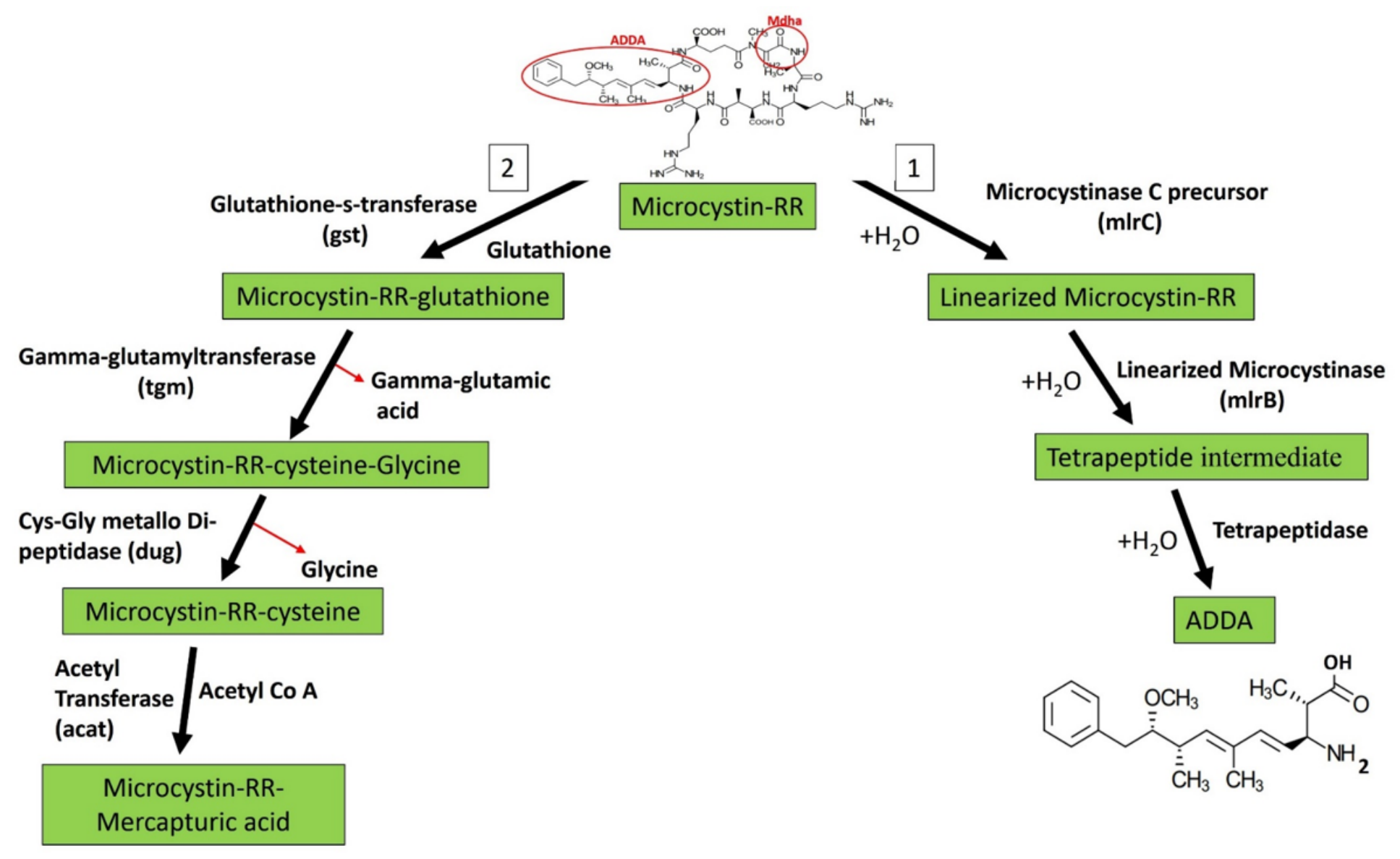

Figure 8

Microcystin degradation pathways found in the Rhizobium genome: Microcystin-RR has two groups, ADDA and Mdha groups are an important part of microcystins, where ADDA allows binding of protein phosphatases 1 and 2A which are the target enzyme. Mdha group covalently binds to the enzyme protein phosphatase's cysteine part. 1. The first step is the cleavage of the peptide ring at the ADDA-arginine bond, followed by subsequent degradation of the linear microcystin-LR product to yield a tetrapeptide intermediate and the ADDA moiety. In Rhizobium, three enzymes jointly denoted as microcystinase operate in a sequential pathway to degrade MC. The first enzyme Microcystinase $\mathrm{C}$ precursor $(\mathrm{mlrC})$ linearizes Microcystin through the cleavage of the peptide ring at the ADDA-arginine bond. The second enzyme linearized microcystinase ( $\mathrm{mlrB}$ ) cleaves this linear intermediate at the alanine-leucine bond, yielding a peptide intermediate of ADDA-Glu-Mdha-Ala. The final enzyme degrades the products formed by the first two enzymes and releases ADDA from the tetrapeptide intermediate. 2. Glutathione (GSH) is a peptide commonly found in the biotransformation of phase II enzymes. Phase II enzyme form a glutathione conjugate, which is a prevalent type of xenobiotic modification, i.e, glutathione-S-transferase (gst). This reaction occurs between the sulfhydryl group of the reduced glutathione and a nucleophilic center on the toxin. Mdha group is responsible for the nucleophilic center of microcystins. GSHconjugation is generally the first sequence of the reaction, eventually producing an $\mathrm{N}$-acetyl-cysteine which is mercapturic acid conjugate can be efficiently removed from the cell. Gammaglutamyltransferase (tgm) is responsible for the enzymatic cleaving of the $y$-glutamic acid group of the 
GSH, forming the intermediate $\gamma$-glutamylcysteine. The glycine of this $\gamma$-glutamylcysteine intermediate is cleaved by a dipeptidase (dug) to form cysteine-conjugate. Which tends to oxidized by Acetyl Transferase-Acetyl Co A (acat) to procedure the mercapturic acid metabolite. This mercapturic acid derivative can removed without difficulty.

\section{Supplementary Files}

This is a list of supplementary files associated with this preprint. Click to download.

- supplementaryfigures2forrevisenewtoupload6may.doc 